B. Schoser ${ }^{1}$, P. Baum ${ }^{2}$, M. Boentert ${ }^{3}$, K. Dillmann ${ }^{4}$, A. Emmer ${ }^{5}$, S. Knauss ${ }^{6}$, E. Enax-Krumova ${ }^{7}$, J. Grosskreutz ${ }^{8}$, A. Güttsches ${ }^{7}$, K. Hellwig ${ }^{9}$, K. Holzapfe ${ }^{10}$, C. Kornblum ${ }^{11}$, H. Lehmann ${ }^{12}$, A. Melms ${ }^{13,14}$, T. Meyer ${ }^{6}$, S. Petri ${ }^{15}$, L. Pilgram ${ }^{16}$, K. Reiners ${ }^{17}$, A. Saak ${ }^{18}$, J. Schäfer ${ }^{18}$, J. Schmidt ${ }^{19}$, C. Schneider-Gold ${ }^{9}$, M. Schons ${ }^{20}$, P. P. Urban ${ }^{21}$, M. Vorgerd ${ }^{7}$, P. Young ${ }^{22}$, S. Zierz ${ }^{5}$

${ }^{1}$ Friedrich-Baur-Institut, Neurologische Klinik und Poliklinik, Ludwig-Maximilians-Universität München, München, Deutschland

${ }^{2}$ Klinik und Poliklinik für Neurologie, Universitätsklinikum Leipzig, Leipzig, Deutschland

${ }^{3}$ Klinik für Neurologie mit Institut für Translationale Neurologie, Universitätsklinikum Münster, Münster, Deutschland

${ }^{4}$ Klinik für Neurologie, Universitätsklinikum Homburg/Saar, Homburg/Saar, Deutschland

${ }^{5}$ Klinik und Poliklinik für Neurologie, Universitätsklinikum Halle, Halle (Saale), Deutschland

${ }^{6}$ Neurologische Hochschulambulanz und ALS-Ambulanz, Charité - Universitätsmedizin Berlin, Berlin, Deutschland

${ }^{7}$ Neurologische Klinik und Poliklinik, BG-Universitätskliniken Bergmannsheil, Ruhr-Universität Bochum, Bochum, Deutschland

${ }^{8}$ Thüringer Neuromuskuläres Zentrum, Klinik für Neurologie, Universitätsklinikum Jena, Jena, Deutschland

${ }^{9}$ Klinik und Poliklinik für Neurologie, Katholisches Universitätsklinikum Bochum, Bochum, Deutschland

${ }^{10}$ Klinik für Neurologie und klinische Neurophysiologie, Universitätsklinikum Augsburg, Augsburg, Deutschland

${ }^{11}$ Klinik und Poliklinik für Neurologie, Sektion für Neuromuskuläre Erkrankungen, Universitätsklinikum Bonn, Bonn, Deutschland

${ }^{12}$ Klinik und Poliklinik für Neurologie, Universitätsklinikum Köln, Köln, Deutschland

${ }^{13}$ Neurologische Praxis Stuttgart, Stuttgart, Deutschland

${ }^{14}$ Zentrum für Neurologie, Universitätsklinikum Tübingen, Tübingen, Deutschland

${ }^{15}$ Klinik und Poliklinik für Neurologie, Medizinische Hochschule Hannover, Hannover, Deutschland

${ }^{16}$ Medizinische Klinik 2, Infektiologie, Universitätsklinikum Frankfurt, Frankfurt, Deutschland

${ }^{17}$ Neurologische Klinik, Hermann-Josef-Krankenhaus Erkelenz, Erkelenz, Deutschland

${ }^{18}$ Klinik und Poliklinik für Neurologie, Universitätsklinikum Dresden, Dresden, Deutschland

${ }^{19}$ Klinik für Neurologie, Neuromuskuläres Zentrum, Universitätsmedizin Göttingen, Göttingen, Deutschland

${ }^{20}$ Klinik I für Innere Medizin, Universitätsklinikum Köln, Köln, Deutschland

${ }^{21}$ Klinik und Poliklinik für Neurologie, Asklepios-Klinikum Hamburg-Barmbek, Hamburg, Deutschland

${ }^{22}$ Klinik für Neurologie MedicalPark, Bad Feilnbach, Deutschland

\title{
SARS-CoV-2/COVID-19 und neuromuskuläre Erkrankungen
}

\author{
Bestandsaufnahme der DGN (Deutsche Gesellschaft für Neurologie) Kommission \\ Motoneuron- und neuromuskuläre Erkrankungen
}

In dieser Bestandsaufnahme werden das aktuell publizierte Wissen zu den primären Auswirkungen einer SARS-CoV-2Infektion/COVID-19-Erkrankung auf das periphere Nervensystem und die Muskulatur sowie zu den sekundären Verschlechterungen präexistenter neuromuskulärer Erkrankungen durch eine SARS-CoV-2-/COVID-19-Erkrankung zusammengefasst und Empfehlungen daraus abgeleitet.

\section{Infektion mit SARS-CoV-2 als neuromuskuläre} Primäraffektion

Häufig fragen Patient*innen mit einer neuromuskulären Erkrankung ihre behandelnden Ärzt*innen nach dem persönli-

\section{Redaktion}

P. Berlit, Berlin

DGNeurologie $2020 \cdot 3(4): 310-320$

https://doi.org/10.1007/s42451-020-00198-2

Online publiziert: 18. Mai 2020

(c) Springer Medizin Verlag GmbH, ein Teil von Springer Nature 2020,

korrigierte Publikation 2020 chen Risiko für einen schweren Verlauf einer COVID-19-Erkrankung. Spezifische Publikationen mit primären Daten zu SARS-CoV-2/COVID-19 und Erkrankungen des peripheren Nervensystems und der Muskulatur liegen bis dato bis auf sehr wenige Kasuistiken aber nicht vor. Daher können im Rahmen dieser Bestandsaufnahme nur aus publizierten COVID19-Kohorten neuromuskuläre Daten extrahiert und kommentiert werden.

Ein erster aktueller Primärdatenzugang wurde uns vom von der DGN (Deutsche Gesellschaft für Neurologie) mitunterstützten „Lean European open survey on SARS-CoV-2 infected patients“ (LEOSS; Infobox 1) zur Verfügung gestellt. Bei LEOSS handelt es sich um ein durch die DGI (Deutsche Gesellschaft für Infektiologie) und die ESCMID („Emerging Infections Task Force“ der „European Society of Clinical Microbiology and Infectious Diseases“) initiiertes multizentrisches Register zur anonymisierten Dokumentation bestätigter SARS-CoV-2-Infektionen, das von den 6 DZG (Deutsche Zentren für Gesundheitsforschung), dem RKI (Robert Koch-Institut) und neben der DGN zahlreichen weiteren medizinischen Gesellschaften unterstützt wird. Die Daten werden dabei nach Beendigung 


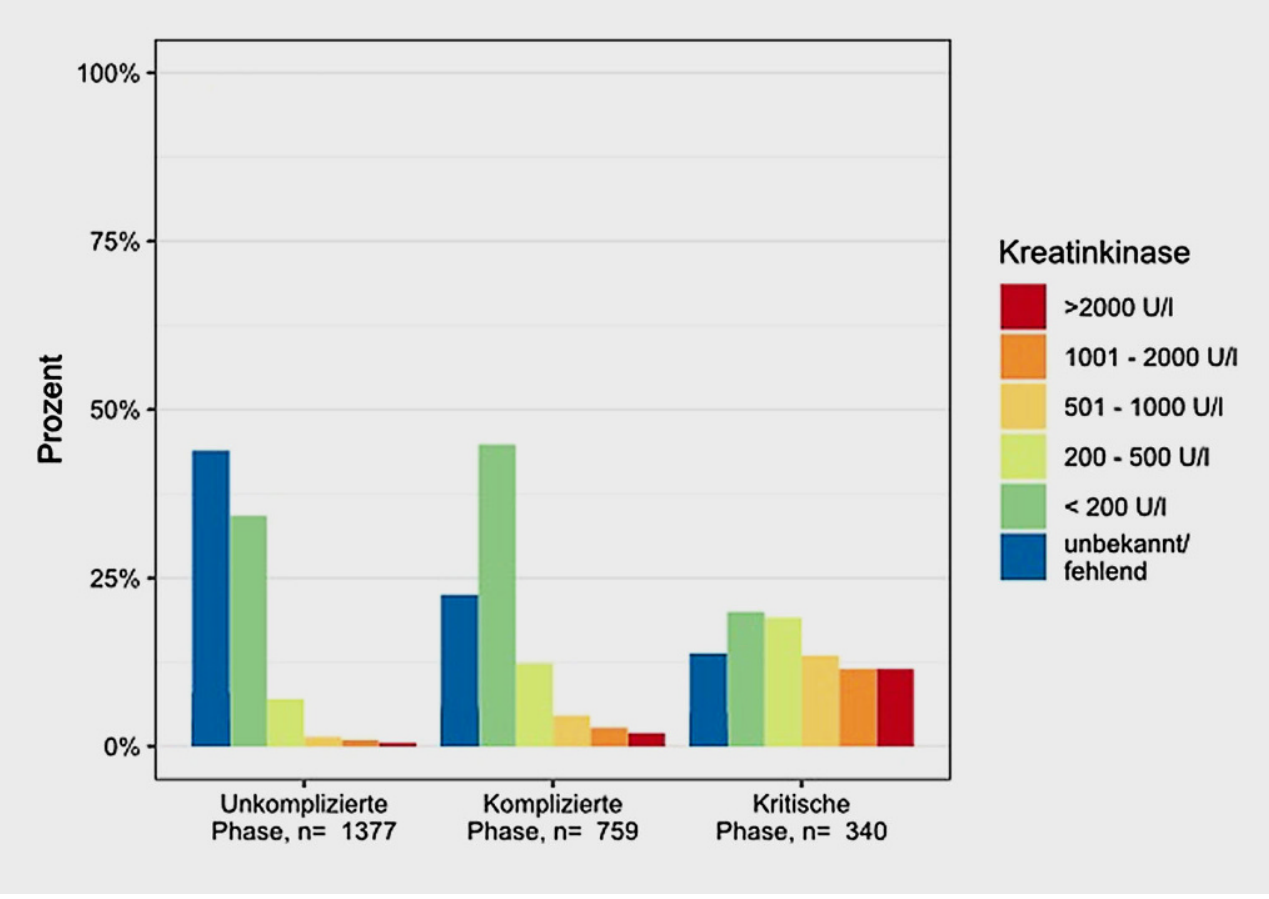

Abb. 1 Kreatinkinase in den verschiedenen Krankheitsphasen, Kriterien der klinischen Phasen bei LEOSS („lean European open survey on SARS-CoV-2 infected patients"; Betrachtung erfolgte getrennt für jede Phase, eine Patient*in kann in verschiedenen Phasen vorkommen; Werte im Fragebogen in den dargestellten Intervallen erhoben. (Aus [54], mit freundl. Genehmigung) der Akutbehandlung retrospektiv erfasst. Perspektivisch werden eine Pseudonymisierung und Verbindung mit Biobanken, Bilddaten und weiteren Patient ${ }^{*}$ innendaten möglich sein. Mittels elektronischem Fragebogen (eCRF) werden soziodemografische und klinische Daten aggregiert über 4 Krankheitsphasen erfasst. Komorbiditäten werden in spezifischen Subfragebögen, die im engen Austausch mit Fachgesellschaften entstanden sind, gezielt adressiert. Die DGN arbeitet an der Konzeption des neurologischen Subfragebogens mit.

Derzeit beteiligen sich aktiv über 85 Universitätskliniken, nichtuniversitäre Krankenhäuser und niedergelassene Kolleg*innen aus Deutschland, aber auch aus Österreich, der Schweiz, Bosnien und Herzegowina, Irland, Spanien, Kanada und der Türkei. Neurologische Vorerkrankungen wie z. B. Myasthenia gravis, multiple Sklerose, neurodegenerative Erkrankungen sowie neurologische Symptome und Komplikationen werden erfasst.

Bis zum 10.05.2020 wurden 2044 COVID-19-Patient ${ }^{*}$ innen vorwiegend im stationären Kontext dokumentiert (815 Frauen/39,9\%). Dabei wurden folgende neuromuskuläre Komorbiditäten erfasst: 1-mal Myasthenia gravis, 4-mal Motoneuronerkrankung und 1-mal eine CIDP (chronische inflammatorische demyelinisierende Polyradikuloneuropathie). Bei 507/1509 (33,6\%, fehlende Werte ausgeschlossen) LEOSS-Register-Patient*innen wurde zum Zeitpunkt des SARS-CoV-2Nachweises mindestens eines der folgenden neurologischen Symptome dokumentiert: Muskelschmerz oder -schwäche, Fatigue, Kopfschmerz, Delir, Meningismus, Geruchs- oder Geschmacksstörung oder sonstige neurologische Symptome. Muskelschmerzen gaben 166/1509 (11\%) und eine Muskelschwäche 144/1509 (9,5\%) an. Die Kreatinkinase (CK) war in der unkomplizierten Phase bei 138/617 (22,4\%) erhöht (>200 U/1), in der komplizierten Phase bei 168/501 (33,5\%) und in der kritischen Phase in der Mehrzahl der Fälle (186/262
[71\%]; fehlende oder nicht bestimmte Werte ausgeschlossen). Ein Überblick der CK-Werte in den verschiedenen Phasen der Infektion ist in Abb. 1 dargestellt.

Eine CK-Erhöhung mit Myalgien als Ausdruck einer Skelettmuskelaffektion wurde bereits in der ersten COVID-19-Kohorte aus Wuhan berichtet [1]. Eine Rhabdomyolyse war bei $0,2 \%$, erhöhte CK-Werte waren bei $13,7 \%$ der 1099 COVID19-Patient ${ }^{*}$ innen nachweisbar [2]. Die Trias aus HyperCKämie, Myalgien und Fatigue stellt die häufigste neuromuskuläre Symptomkonstellation dar, die Prävalenz schwankt zwischen 40 und $70 \%$ der COVID-19-Erkrankten in den unterschiedlichen Kohorten. Bei bis zu $30 \%$ der Erkrankten findet sich die CKErhöhung im Median bereits nach 1-2 Tagen [1-5]. Ob es sich dabei um eine SARS-CoV-2-assoziierte Myositis oder Vaskulitis handelt, bleibt unklar, da keine histologischen Untersuchungen publiziert sind [6]. Aus älteren Studien war ersichtlich, dass sich SARS-ähnliche virale Partikel und SARS-CoVRNA (RNA: Ribonukleinsäure) in T-Lymphozyten unterschiedlicher Organe nachweisen lassen. Die Rezeptorbindungsdomäne der Spikeproteine weist zwischen SARS-CoV-2 und SARS$\mathrm{CoV}$ eine hohe Übereinstimmung auf. Somit kommt es durch die SARS-CoV-2-Infektion hochwahrscheinlich neben der Aktivierung des Zelltods durch eine Fas- und Fas-Liganden-Interaktion und einer Tumornekrose-Faktor-vermittelten Apoptose auch zu einer direkten Infektion von T-Lymphozyten [6].

\section{Konsequenzen der SARS-CoV-2-Pandemie für die neuromuskuläre Diagnostik}

Aufgrund der hohen Kontagiosität von SARS-CoV-2 wurde national und international auf apparativ-technische Untersuchungen wie EMG (Elektromyographie), Muskel-MRT (MRT: Magnetresonanztomographie) oder auch eine Muskelbiopsie überwiegend verzichtet [7]. Bei spezifischen Fragestellungen (z. B. floride Myositis vs. metabolische Myopathie) kann auch auf 
der Intensivstation eine Bedside-EMG unter Beachtung der Hygienemaßnahmen durchgeführt werden. Eine MRT der Muskulatur erscheint aufgrund des hohen Aufwands, verbunden mit einem erhöhten Risiko bei intensivpflichtigen Patient ${ }^{*}$ innen und der erforderlichen Schlussdesinfektion des MRT-Geräts, in der Risiko-Nutzen-Abwägung aktuell weiterhin nicht indiziert. Bei dringendem Bedarf einer bildgebenden Untersuchung kann eine Muskelsonographie erfolgen. Aktuell wird in den neuromuskulären Zentren eine Wiedereröffnung der ambulanten und stationären Betreuung initiiert, die diese oben gennannten Einschränkung den Umständen angepasst überwindet.

\section{Patient*innen mit präexistenten Motoneuron- und neuromuskulären Erkrankungen}

Patient*innen mit neuromuskulären und Motoneuronerkrankungen sind aufgrund deren Progredienz und Komplexität auf eine engmaschige multidisziplinäre und institutionsübergreifende dauerhafte Versorgung angewiesen. Bei Beteiligung der Atem- und Schluckmuskulatur, durch Einnahme immunsuppressiver Medikamente und durch Einschränkung der medizinischen und pflegerischen Versorgung bei Barrieremaßnahmen ergibt sich eine Gefährdung. In der Regel wird sich auch diese Gruppe von Patient*innen von einer COVID-19-Erkrankung erholen. Sollte jedoch eine wochenlange invasive Beatmungsbehandlung erforderlich sein, kann dies zu einer dauerhaften Beatmungspflichtigkeit führen. Gesicherte Daten hierzu gibt es aber bisher nicht.

\section{Amyotrophe Lateralsklerose und Motoneuronerkrankungen}

Bisher existieren keine spezifischen Publikationen zum individuellen Risiko und Verlauf einer Motoneuronerkrankung unter SARS-CoV-2/COVID-19. Gemäß den publizierten Konsensusempfehlungen einer europäischen Expertengruppe [8] sind ALS-Patient*innen (ALS: amyotrophe Lateralsklerose) mit respiratorischer Insuffizienz und/oder rascher Krankheitsprogression generell als Risikogruppe für eine SARS-CoV-2-Infektion mit schwerem Verlauf anzusehen. Die Konsensusempfehlungen der europäischen Expertengruppe beinhalten Maßnahmen zur Vermeidung sozialer Kontakte und zu Hygienemaßnahmen. Digital unterstützte Versorgungsnetzwerke und Fallmanagementplattformen sowie damit verbundene Applikationen (sog. Apps) können zum Konzept der sozialen Distanzierung - unter Beibehaltung der Versorgungsprozesse - beitragen. Spezifische Empfehlungen zur Atemtherapie und Dysphagie finden sich am Ende dieses Berichts.

\section{Hereditäre Myopathien}

Auch für hereditäre Myopathien existieren keine Daten zu SARS-CoV-2/COVID-19. Das Risiko für den Verlauf einer schweren COVID-19-Erkrankung hängt von der jeweiligen Schwere der präexistenten neuromuskulären Grunderkrankung, weiteren Vorerkrankungen und einer Beteiligung von Herz- und Atemmuskulatur ab. Patient*innen mit einer milden Erkrankung ohne Beteiligung der Atemmuskulatur und ohne immunsuppressive Therapie, die keine weiteren Vorerkrankungen aufweisen, haben kein höheres Risiko der Entwicklung einer schweren COVID-19-Erkrankung im Vergleich zur Normalbevölkerung [9]. Patient ${ }^{\star}$ innen mit einer bereits vorhan- denen Schwäche der Atemhilfsmuskulatur, insbesondere bei Beatmungspflichtigkeit, haben hingegen ein höheres Risiko für einen schwereren COVID-19-Verlauf. Dies betrifft in gewissem Grad auch Patient*innen mit einer Dysphagie und Schwäche der Kaumuskulatur [9]. Bei bereits fortgeschrittenem Stadium ist es wahrscheinlich, dass sich die neuromuskuläre Erkrankung durch SARS-CoV-2/COVID-19 verschlechtert und das klinische Ausgangsniveau vor der Infektion nach Überwinden der akuten Erkrankung nicht mehr erreicht wird [9]. Daher sollten alle Patient*innen mit einer Myopathie bezüglich einer Verschlechterung ihrer respiratorischen Funktion und ihrer neuromuskulären Erkrankung ggf. auch telemedizinisch betreut werden [9].

\section{Mitochondriale Erkrankungen}

Bei mitochondrialen Erkrankungen ist die krankheitsdefinierende Störung der mitochondrialen Energiegewinnung über die Atmungskette als möglicher Risikofaktor für einen schwereren Verlauf von COVID-19 zu betrachten. Im Rahmen systemischer inflammatorischer Prozesse kann bei bereits beeinträchtigter Atmungskettenfunktion rasch eine metabolische Dekompensation auftreten [10]. Viren können zentrale mitochondriale Funktionen beeinflussen und so die Zellmaschinerie zugunsten viraler Proliferation manipulieren $[11,12]$. Unter anderem sind Effekte auf Mitophagie, Autophagie und Regulationsstörungen mitochondrialer Proteine beschrieben [13]. Das Betacoronavirus SARS-CoV verursacht durch die spezifische Gensequenz ORF9 („open reading frame“) eine mitochondrial getriggerte Störung der angeborenen Immunität, der antiviralen Signalwege und der mitochondrialen Dynamik mit mitochondrialer Elongation. Laut bioinformatischer Analysen ist eine Herunterregulierung („downregulation“) mitochondrialer Proteine durch SARS-CoV-2 zu postulieren [14].

Muskulatur. Mitochondriale Erkrankungen können mit restriktiven Ventilationsstörungen, ggf. in Kombination mit einer thorakolumbalen Skoliose, einhergehen. Ob eine Störung der oxidativen Phosphorylierung generell eine Prädisposition für einen schweren COVID-19-Verlauf und eine muskuläre Mitbeteiligung darstellt, ist unklar. Aufgrund der bereits beeinträchtigten Atmungskettenfunktion ist ein ungünstiger Effekt einer Hypoxie naheliegend.

Herz. Patient*innen mit kardialen Vorerkrankungen werden als Risikopatienten für schwerere COVID-19-Verläufe angesehen. Kardiale Symptome können bei vorbestehender kardialer Beteiligung im Rahmen einer mitochondrialen Erkrankung exazerbieren. Daher sollte bei mitochondrialen Erkrankungen unter COVID-19 ein intensiviertes kardiales Monitoring erfolgen $[1,15]$.

Mitochondriale Erkrankung und Diabetes. Ein vorbestehender Diabetes mellitus bei mitochondrialer Erkrankung wird als Risiko für einen schweren COVID-19-Verlauf angesehen, und ein nicht diagnostizierter Diabetes kann sich demaskieren. Bei diabetischer Stoffwechsellage kann COVID$19 \mathrm{zu}$ einer hyperosmolaren Hyperglykämie und Ketoazidose führen [16]. Spezifische Therapieempfehlungen der DDG (Deutsche Diabetes Gesellschaft) und anderer Diabetesverei- 
Hier steht eine Anzeige.

Springer 
nigungen sind publiziert [17]. Bei COVID-19 ist besonders auf die Einstellung des Blutzuckerspiegels zu achten und nach einem latenten Diabetes mellitus zu fahnden.

Gastrointestinale Symptome. Im Rahmen von COVID-19 sind gastrointestinale Beschwerden häufig [18]. Bei mitochondrialen Erkrankungen sollten ggf. bei nichtkontaktfähigen, intensivpflichtigen Patient*innen die Serumlaktatspiegel überwacht werden. Die Erkrankung COVID-19 kann zu einem deutlichen Gewichtsverlust führen, dies sollte bei mitochondrialen Erkrankungen besonders beachtet werden. Fasten sollte vermieden werden, um eine metabolische Krise zu verhindern.

„Stroke-like episodes" und Epilepsie. Bei bestimmten mitochondrialen Erkrankungen treten schlaganfallähnliche Episoden auf, die Ausdruck einer zerebralen metabolischen Dekompensation bei fokaler epileptischer Aktivität sind, die aggressiv antikonvulsiv behandelt werden sollte. Schlaganfallähnliche Episoden können im Sinne einer metabolischen Krise durch akute Infekte und/oder Fieber getriggert sein. Diese katabolen Zustände sollten möglichst vermieden und Fieber zügig gesenkt werden [19].

Auch das Risiko für epileptische (Rezidiv-)Anfälle kann durch COVID-19 gesteigert werden, sodass hieran erkrankte Patient*innen mit mitochondrialer Zytopathie und Epilepsie optimiert antikonvulsiv behandelt werden sollten [20].

Medikamente und mitochondrialer Stoffwechsel (Auswahl). Gemäß einem internationalen Konsensusstatement zur Sicherheit verschiedener Arzneimittel bei mitochondrialen Erkrankungen [21] finden sich im Zusammenhang mit Infektionskrankheiten folgende relevante Substanzen gelistet: Propofol ist für die kontinuierliche Gabe zur Sedierung mit Vorsicht zu verwenden. Es gibt Hinweise auf eine Störung der Atmungskette und der $\beta$-Oxidation der Fettsäuren. Das Monitoring der Serum-CK und des Serummyoglobins wird empfohlen. Die kurzzeitige Anwendung von Propofol, z.B. zur Narkoseeinleitung, ist möglich. Eine Alternative stellt Midazolam dar.

Die Anwendung von Aminoglykosiden sollte aufgrund ihrer Ototoxizität vermieden werden.

Tetrazykline wirken auf die bakterielle Proteinbiosynthese; die Beeinträchtigung der mitochondrialen Proteinbiosynthese ist denkbar, daher ist ihr Einsatz nur bedingt zu empfehlen.

Bei therapierefraktärer Epilepsie kann Valproinsäure eingesetzt werden, wenn eine POLG-Mutation (POLG: DNA-Polymerase gamma [DNA: Desoxyribonukleinsäure]) ausgeschlossen wurde.

\section{Endokrine, toxische und metabolische Myopathien}

Spezifische Daten zu COVID-19 und dem Verlauf endokriner, toxischer und metabolischer Myopathien liegen nicht vor. Dysthyreote Myopathien wurden in einer deutschen Kohorte hospitalisierter COVID-19-Patient ${ }^{*}$ innen mit und ohne ARDS („acute respiratory distress syndrome“) nicht gefunden [22].

Eine Steroidmyopathie kann durch eine gesteigerte Hormonsekretion der Nebennierenrinden, ACTH (adrenokortikotropes Hormon bzw. Kortikotropin) oder durch Glukokortikoidtherapie hervorgerufen werden. Intensivmedizinisch werden
Glukokortikoide in unterschiedlicher Indikation eingesetzt. Eine steroidinduzierte Muskelschwäche ist innerhalb der ersten 4 Wochen in der Regel nicht zu erwarten und somit auch nicht in der COVID-19-Akutphase. Bei pulmonal vorerkrankten $\mathrm{Pa}$ tient*innen mit schwerer COVID-19 kann jedoch eine längerfristige hochdosierte Glukokortikoidtherapie erforderlich sein, sodass eine akute Steroidmyopathie oder CIM („critical illness myopathy") möglich sind. Glukokortikoide sollten daher kurz und in geringster Dosis eingesetzt werden.

Exazerbationen toxischer Myopathien sind sowohl durch das Virus selbst als auch durch eingesetzte Virostatika möglich. Statine werden wegen ihres lungenprotektiven antiinflammatorischen Effektes als Adjuvans zur Behandlung schwerer COVID-Fälle aktuell empfohlen [23], dem stehen jedoch potenziell toxische Effekte mit Rhabdomyolyse gegenüber. Es gibt keine Evidenz, eine aus kardioneurovaskulärer Indikation begonnene Statintherapie unter COVID-19 abzusetzen. Patient*innen mit bekannter toxischer Myopathie müssen bei akuter, signifikanter Zunahme ihrer Myalgien und Beschwerden auf eine SARSCoV-2-Infektion untersucht werden.

Bei Patient*innen mit metabolischen Myopathien (Glykogenosen, Fettsäureoxidationsdefekte, Mitochondriopathien) können durch Fasten, Stress und Infektionen gehäuft Episoden mit Myalgien, Muskelschwäche und Myoglobinurie auftreten. Bei bis zu $25 \%$ der Patient*innen mit einer Myoglobinurie ist die Entwicklung einer Niereninsuffizienz möglich, welche COVID19 aggravieren kann.

Beim M. Pompe, mit bei 1/3 der Betroffenen vorhandener Ateminsuffizienz, sind ein temporäres Pausieren der Enzymersatztherapie oder die Gabe der Infusionen zuhause zu erwägen. Da eine Verschlechterung der Vitalkapazität und Gehfähigkeit beim Pausieren möglich ist, sollte individuell eine Nutzen-Risiko-Abwägung erfolgen. Bisher wurde ein Einzelfall einer Kombination von Rhabdomyolyse und COVID-19 berichtet [24].

\section{Toxische Myopathien als mögliche Nebenwirkung derzeitiger COVID-19-Studienmedikamente}

Unter den aktuell 795 gelisteten Studien zur COVID-19 rekrutieren 352 mit verschiedensten Therapieansätzen (Stand: 23.04.2020, www.clinicaltrials.gov). Dabei lassen sich 3 Wirkmechanismen unterteilen:

1. Inhibitoren der viralen RNA-Synthese,

2. Antivirale Entry-Inhibitoren und

3. Immunmodulatoren bzw. -therapien.

Lopinavir/Ritonavir werden z. B. in der europäischen DISCOVERY-Studie (Remdesivir, Lopinavir/Ritonavir, Chloroquin) als Virostatikum eingesetzt. In Einzelfällen wurden Rhabdomyolysen berichtet, meist jedoch bei Komedikation mit Statinen oder Makroliden. Unter Remdesivir wurden in den Studien zu Ebola keine Myopathien beobachtet. Chloroquin wird bei SARS-CoV-2-Infektion eine direkte antivirale und antiinflammatorische Wirkung zugeschrieben.

Bezüglich möglicher neuromuskulärer Komplikationen sind besonders Chloroquine, oft in Kombination mit Azithromycin, hervorzuheben [25-27]. Chloroquinmyopathien sind klinisch oft inapparent, gehen mit einer CK-Erhöhung und histologisch Vakuolen mit dem Nachweis sog. „curvilinear bodies“ einher. Eine Chloroquinmyopathie entwickelt sich meist erst nach 6-monatiger Therapie, was im Rahmen der COVID-19- 
Behandlung kaum erreicht werden wird. Nach Beendigung der Chloroquingabe sind die muskulären Symptome meist deutlich oder komplett reversibel [25-27]. Häufigere und gefürchtete Nebenwirkungen von Chloroquinen sind Kardiotoxizität, sensible Neuropathie, myasthenes Syndrom und eine irreversible Retinopathie. Unter Berücksichtigung der nur geringen Wirksamkeit sollte der Chloroquineinsatz bei COVID-19 in individueller Nutzen-Risiko-Abwägung kritisch diskutiert werden.

\section{Neuropathien}

In einer retrospektiven Fallserie mit 214 stationären COVID19-Patient*innen fanden sich bei 78 neurologische Manifestationen. Hirnnervensymptome wie Hypogeusie, Hyposmie oder Polyneuritis cranialis hatten $19(8,9 \%)$. Geschmacks- $(5,6 \%)$ und Geruchsstörungen (5,1\%) traten am häufigsten auf [3].

Diabetes mellitus, chronische Leber-, Nieren- und Lungenerkrankungen, hämatologische Erkrankungen, Tumorerkrankungen, Organtransplantationen sowie die Alkoholerkrankung sind häufig mit einer Polyneuropathie assoziiert. Die hiervon betroffenen Patient ${ }^{*}$ innen haben ein erhöhtes Risiko für einen schwere COVID-19-Erkrankung [1-5]. Es gibt Hinweise auf eine erhöhte Inzidenz und Schwere von SARS-CoV-2Infektionen bei Diabetespatienten aufgrund des verstärkten Eindringens des Virus in die Zelle und der beeinträchtigten Immunantwort [28]. Daher ist prophylaktisch eine optimierte Blutzuckerspiegeleinstellung wichtig [17, 28]. Eine autonome Neuropathie kann ein zusätzliches Risiko für einen schwereren COVID-19-Verlauf und eine erhöhte Mortalität darstellen. Betroffene sollten auf eine Störung des autonomen Nervensystems mit gestörter Pupillenlichtreaktion, trophischen Störungen der Haut (Haarverlust, Temperaturveränderungen, Palmar-/Plantarerythem, Ödeme, Ulkus) Osteoarthropathie, Hypo-/Anhidrose, Sinustachykardie und orthostatische Hypotonie untersucht werden. Verschiedene Chemotherapeutika, Antibiotika, Immun-Checkpoint-Inhibitoren, Proteaseinhibitoren und Chloroquine können exotoxische Neuropathien verursachen, die sich ggf. unter einer SARS-CoV-2-Infektion verschlechtern.

Bei Patient*innen mit einer SARS-CoV-2-Infektion ist die Wahrscheinlichkeit, eine bereits bestehende Polyneuropathie mit Lopinavir/Ritonavir oder Chloroquin $\mathrm{zu}$ induzieren oder zu verschlimmern, vermutlich niedrig. Für eine Vielzahl rein sensibler oder sensibel betonter Neuropathien ist die Gefährdung als eher gering einzuschätzen. Für die unterschiedlichen erblichen Neuropathien ist bislang vollkommen unklar, wie sich eine SARS-CoV-2-Infektion auswirkt. Ein erhöhtes Risiko für die Entwicklung einer CIP („critical illness polyneuropathy“) bei Patient*innen mit erblichen Neuropathien wurde bislang nicht beobachtet.

\section{Erworbene Intensivstations-Muskelschwäche (ICUAW), Critical-IIIness-Neuropathie (CIP) und Criticall-Illness- Myopathie (CIM)}

Aktuell werden in Deutschland 1576 CODID-19-Patient*innen intensivmedizinisch behandelt, davon sind 1063 beatmet. Von den insgesamt 10.929 intensivmedizinisch behandelten COVID-19-Patient ${ }^{*}$ innen sind 2032 (18,9\%) verstorben [55]. Die Rate schwerer COVID-19-Erkrankungen mit intensivmedizi- nischer Behandlungsnotwendigkeit und invasiver Beatmung beträgt nach einer aktuellen Publikation 3,2\% [29]. Schwere Pneumonien mit intensivmedizinischer Behandlung führen zur sog. ICUAW („ICU-acquired weakness“ [ICU: Intensivstation]), einem Krankheitsbild, bei dem die CIP („critical illness polyneuropathy“) und CIM („critical illness myopathy“) ineinandergreifen. Beide kommen nebeneinander vor und lassen sich rein klinisch nicht unterscheiden [30]. Eine CIP und CIM sind bei schwer erkrankten COVID-19-Patient*innen wahrscheinlich und wurden bereits bei anderen Coronavirusinfektionen beschrieben [29-33]. In einer systematischen Übersicht von 31 Studien wurde über eine mediane Prävalenz von $43 \%$ berichtet [33]. Die Inzidenz nimmt mit der Schwere und Dauer der kritischen Erkrankung zu und erhöht sich durch weitere Faktoren wie Sepsis, Multiorganversagen, Hyperglykämie, parenterale Ernährung und bestimmte Medikamente (Sedativa, Antibiotika, Kortikosteroide, Muskelrelaxanzien; [30]). Bei intensivpflichtigen COVID-19-Patient ${ }^{*}$ innen treten sehr häufig frühmotorische Defizite auf, die auf eine CIP und CIM zurückzuführen sind. Das klinische Bild ist geprägt durch die Schwäche und Atrophie der gesamten Muskulatur, inklusive der Atmungsmuskulatur, und Empfindungsstörungen [31, 32]. Eine reduzierte Muskelmasse führt zu einem schlechteren Outcome bei „critical illness“. Für die erhöhte Mortalität werden ein veränderter Muskelmetabolismus, ein mitochondriales Energiedefizit und ein chronisch-kataboler Status angeführt [34].

Beide, CIP und CIM, führen zu Problemen bei der Entwöhnung vom Respirator, prolongieren die Intensivbehandlung und erhöhen die Mortalität [34-36]. Schon eine leicht reduzierte Muskelkraft hat Einfluss auf die Langzeitmortalität [37]. Ehemals kritisch kranke Patient*innen klagen über persistierende Sensibilitätsstörungen, Dysästhesien und motorische Defizite, die mit einer reduzierten Lebensqualität einhergehen [38, 39]. Zur Prävention sollten Hyperglykämien vermieden, die parenterale Ernährung über die erste Woche auf der Intensivstation hinaus verschoben und die Sedierung minimiert werden [30]. Multiprofessionelle Maßnahmen zur Rehabilitation sollen schon während der Akutversorgung begonnen werden.

\section{Triage neuromuskulärer Patient*innen}

Hinsichtlich einer Triage für intensivmedizinische Therapien ist anzumerken, dass eine neuromuskuläre Erkrankung mit einem stabilen permanenten Defizit als prognostisch günstige Vorerkrankung einzustufen ist. Dies gilt ebenso auch für $\mathrm{Pa}$ tient*innen mit neuromuskulären Erkrankungen ohne zusätzliche kardiorespiratorische Beteiligung. Eine intensivmedizinische Behandlung steht auch progredient neuromuskulär Erkrankten mit z.B. einer Motoneuronerkrankung zu, die eine dauerhafte Beatmungstherapie wünschen.

\section{Guillain-Barré-Syndrom (GBS)}

Die Ergebnisse mehrerer Fallberichte und kleinerer Fallserien legen nahe, dass auch SARS-CoV-2 einen Trigger für ein Guillain-Barré-Syndrom darstellen kann [40-42]. In der bisher größten Fallserie sind 5 Patient ${ }^{*}$ innen beschrieben, die innerhalb von 5-10 Tagen nach Symptombeginn von COVID-19 ein GBS entwickelten [41]. Von diesen 5 Patient $^{*}$ innen mussten 3 maschinell beatmet werden, wobei unklar blieb, ob die Beat- 
Infobox 1 Internetlinks

- https://leoss.net

- www.clinicaltrials.gov

mung wegen des GBS oder wegen der respiratorischen Infektion notwendig wurde. Auffällig ist das kurze Intervall zwischen den Symptomen der SARS-CoV-2-Infektion und dem Beginn des GBS.

Weiterhin wurden 2 Patient*innen mit Miller-Fisher-Syndrom (MFS) und 2 weitere Patient ${ }^{*}$ innen mit einer Ophthalmoparese berichtet $[43,44]$.

Als Empfehlungen muss gefolgert werden: Die SARS-CoV2-Diagnostik sollte bei Verdacht auf ein GBS bzw. MFS erfolgen; bei COVID-19 mit Beatmungspflichtigkeit bzw. prolongierter Respiratorentwöhnung muss ein GBS als Ursache ausgeschlossen werden. Da das GBS durch Autoantikörper vermittelt wird, birgt ein experimentelles Therapiekonzept mittels sog. passiver Immunisierung durch Übertragung von IgG (Immunglobulin G) genesender COVID-19-Patient*innen auf schwer erkrankte COVID-19-Patient*innen das theoretische Risiko einer Auslösung eines GBS.

\section{Chronische inflammatorische demyelinisierende Polyneuropathie (CIDP)}

Es gibt keine gesicherten Erkenntnisse zur möglichen Interaktion hinsichtlich Krankheitsentstehung oder Beeinflussung einer CIDP durch SARS-CoV-2/COVID-19. Viele CIDP-Patient*innen werden mit immunmodulatorischen bzw. immunsuppressiven Substanzen behandelt. Während Erstere unkritisch sind, besteht bei Letzteren ein theoretisches Risiko für Infektionen. Prinzipiell sollte bei stabilen CIDP-Patient*innen grundsätzlich die Indikation für eine Weiterführung der immunsuppressiven Therapie regelmäßig überprüft werden.

\section{Myasthenie und Lambert-Eaton-Syndrom}

Bisher liegen keine Berichte vor, dass eine Myasthenia gravis (MG) bzw. ein Lambert-Eaton-Myasthenes-Syndrom (LEMS) durch eine SARS-CoV-2-Infektion getriggert wurden. Befürchtungen, dass immunsupprimierte MG- und LEMS-Patient ${ }^{*}$ innen ein erhöhtes Risiko hätten, sich mit SARS-CoV-2 $\mathrm{zu}$ infizieren, konnten bisher nicht bestätigt werden. Somit gelten die Empfehlungen, eine bis dato durchgeführte symptomatische (Pyridostigmin und 3,4-Diamino-Pyridin/Fampridin) Behandlung und die immunmodulierende Therapie einschließlich der Gabe von Steroiden oder Eculizumab fortzuführen [45, 46]. Die Gabe von Rituximab oder die Einleitung einer oralen Langzeitimmunsuppression können in Abhängigkeit vom klinischen Zustand des Betroffenen und der Anamnese ggf. verschoben werden. Für MG-/LEMS-Patient*innen gelten die gleichen Risikoabwägungen und Impfempfehlungen (Influenza- und Pneumokokkenimpfung) wie für Gesunde der gleichen Altersgruppe bei nur geringen Impfrisiken. Der sog. Zytokinsturm bei COVID-19 scheint unter Immunsuppression eher geringer ausprägt. Bei schwerer COVID-19 mit ARDS kann es zu einer Komplementaktivierung kommen [47]. Eine Behandlung mit Eculizumab sollte daher nicht unterbrochen werden, da durch die Hemmung der terminalen Komplementaktivierung das Ausmaß des Zytokinsturms und die Gewebe-

\section{Infobox 2 Fall-Vignette}

SARS-CoV-2-assoziierte Myositis mit Differenzialdiagnose einer SARSCoV-2-assoziierten Rhabdomyolyse

Ein zuvor Gesunder suchte aufgrund einer akuten proximalen myalgischen Paraparese mit Sturz die Notaufnahme auf. Dort fanden sich eine moderate Paraparese der Hüftmuskulatur (Kraftgrad 3/5 MRCS) und eine massive HyperCKämie von 25.384U/I (Norm< 195 U/I), das CRP (C-reaktives Protein) war auf $54 \mathrm{mg} / \mathrm{l}$ erhöht, und es lag eine Lymphozytopenie vor. Der Patient war afrebril und ohne Hinweise auf einen Atemwegsinfekt. Nach 4 Tagen fieberte der Patient bis $39^{\circ} \mathrm{C}$. Eine neue SARS-CoV-2-PCR (PCR: Polymerasekettenreaktion) war negativ. Am 5. Tag zeigte eine CT (Computertomographie) des Thorax eine bilaterale Milchglastrübung beider Unterlappen, und am Tag 7 benötigte der Patient Sauerstoffgaben. Die SARS-CoV-2-PCR war erneut negativ. Ein Muskel-MRT ergab ein bilaterales Ödem im M. obturatorius und M. quadriceps femoris, kompatibel mit einer Myositis. Myositisspezifische Antikörper waren negativ. Auch eine dritte SARS-CoV-2-PCR am Tag 10 war negativ. Aufgrund der pulmonalen Verschlechterung wurde der Patient auf die Intensivstation verlegt. Erst eine SARS-CoV-2-PCR aus der Bronchiallavage am Tage 11 ergab die Bestätigung der COVID-19-Diagnose. Der weitere klinische Verlauf und das Outcome sind nicht beschrieben [48].

Kritisch muss hier angemerkt werden, dass keine histologische Diagnosesicherung erfolgte, sodass alternativ eine Rhabdomyolyse durch SARS-CoV-2 zu diskutieren ist. Hierzu waren der klinische Verlauf und der MRT-Befund ebenso passend. Der CT-Befund des Thorax war zudem mit einer frühen COVID-19-Manifestation kompatibel [49]. Wichtig ist, dass eine Lungenbeteiligung, wie sie als "interstitial lung disease“ (ILD) beim Antisynthetasesyndrom oder der Dermatomyositis vorkommen kann, sowohl klinisch als auch radiologische eine wichtige Differenzialdiagnose einer durch COVID-19 bedingten pulmonalen Schädigung darstellt. Eine parainfektiöse Genese der Muskelschädigung durch immunvermittelte Mechanismen ist bei SARS-CoV-2 wahrscheinlich. Unterstützt wird diese Hypothese durch eine histopathologische Aufarbeitung von 8 Fällen im Rahmen der SARS-Epidemie im Jahr 2003 [50]. Hierbei fanden sich Muskelfasernekrosen und Muskelfaseratrophien. Die Autoren diskutierten eine CIM sowie eine immunvermittelte Muskelfasernekrose als Ursache. Virale Partikel konnten im Muskel nicht identifiziert werden.

schädigung in der Lunge begrenzt werden. Prinzipiell können Infektionen und somit auch eine SARS-CoV-2-Infektion eine Myasthenie oder ein LEMS verschlechtern, wobei hinsichtlich der Ursache einer respiratorischen Insuffizienz möglicherweise keine klare Ursachentrennung erfolgen kann. Bei erhöhten CKWerten, Myalgien und persistierender neuer Muskelschwäche sollte die Möglichkeit einer SARS-CoV-2-getriggerten Myositis (s. unten) bedacht werden. Die mancherorts propagierte Kombination von Azithromycin und Chloroquin sollte nicht unkritisch eingesetzt werden, da sich eine Myasthenie hierunter verschlechtern kann. Bei vermuteter myasthener Krise können und sollten nach jetzigem Kenntnisstand alle üblichen rasch wirksamen therapeutischen Maßnahmen einschließlich Plasmaphereseverfahren und der Gabe von Immunglobulinen unter Beachtung der kardialen Situation durchgeführt werden.

\section{Inflammatorische Myopathien}

Es liegt bisher nur ein einzelner Fallbericht zum Auftreten einer möglichen Myositis im Rahmen einer SARS-CoV-2-Infektion vor (s. Fall-Vignette, Infobox 2; [48]). Die Therapieempfehlungen im Zusammenhang mit COVID-19 entsprechen denen bei Myasthenie/LEMS. 


\section{Allgemeine Empfehlungen für neuromuskuläre Patient*innen}

In Deutschland werden neuromuskuläre Patient*innen flächendeckend in neuromuskulären Zentren betreut. Dabei werden telefonische Arztkontakte, videogestützte Konsultationen und direkte persönliche Konsultationen unter Wahrung des Abstandsgebots mit teilstationären und stationären Behandlungsangeboten abgestimmt, um die notwendige Versorgungsdichte in Zusammenarbeit mit digital und internetgestützten Koordinierungs- und Versorgungsdiensten zu gewährleisten. Es besteht ein enger Austausch zu den Selbsthilfeorganisationen wie der DGM (Deutsche Gesellschaft für Muskelkranke e. V.) u.a.

Die WMS („World Muscle Society“) stellte im April 2020 eine erste umfassende Konsensusempfehlung für SARS-CoV2/COVID-19 und neuromuskuläre Erkrankungen online [51].

\section{Allgemeine Empfehlungen bei respiratorischer Verschlechterung}

Beim Auftreten von COVID-19-verdächtigen Symptomen nach Kontakt mit einer potenziell infizierten Person, bei respiratorischer Dekompensation beatmeter Patient ${ }^{*}$ innen oder allgemein bei neu aufgetretenen respiratorischen Symptomen werden eine telefonische oder telemedizinische Kontaktaufnahme mit dem behandelnden Arzt sowie ggf. dem Beatmungspflegedienst empfohlen. Die Entscheidung über die Einleitung einer ambulanten oder stationären Diagnostik und Therapie wird individuell getroffen. Bei Notwendigkeit eines Transports von Patient*innen mit nichtinvasiver oder invasiver Beatmung in ein Krankenhaus müssen entsprechende Filtersysteme verwendet werden. In einer Stellungnahme der „European Academy of Neurology“ werden spezifische Maßnahmen für einen Krankentransport eines infizierten beatmeten Betroffenen vorgeschlagen (nichtbelüftete Maske mit einem Bakterien-VirenFilter am Geräteausgang, Filter zwischen Maske und Geräteschlauch). Bei Nutzung eines mechanischen Insufflator/ Exsufflators sollen bei infizierten Patient*innen intensivierte Hygienemaßnahmen eingehalten werden (Tragen einer FFP2-Maske [FFP: „filtering face piece“], von Schutzbrillen, Handschuhen und Schutzkleidung durch die durchführende Pflegeperson, sofortige Entsorgung aller biologischen Materialien, Dusche der Pflegeperson nach der Versorgung des Betroffenen).

Auch die Empfehlungen der WMS beinhalten weitgehend vergleichbare Regeln zur sozialen Distanzierung bis hin zur häuslichen Quarantäne für Hochrisikopatient*innen, sorgfältigen Beachtung von Hygienemaßnahmen (Händewaschen, Hand- und Oberflächendesinfektion, Weiterführung von Physiotherapien per Videosprechstunde). Hier wird für nicht im Haushalt lebende Pflegepersonen das Tragen einer FFP2Schutzmaske angeraten. Außerdem wird auf die Notwendigkeit von Notfallplänen und ausreichender Bevorratung von Medikation und Pflegemitteln für den Fall des Ausfalls einer Pflegeperson durch Quarantäne oder eine Quarantänephase des Erkrankten hingewiesen, um eine Krankenhauseinweisung nach Möglichkeit zu vermeiden.

In spezifischen Empfehlungen der „World Muscle Society“ zu neuromuskulären Patient ${ }^{*}$ innen mit Heimbeatmung, die so auch für heimbeatmete ALS-Patient ${ }^{*}$ innen gelten müssen, werden die Notwendigkeit von Bakterien-Viren-Filtern betont, die bei Nichtvorhandensein in Rücksprache mit dem Hersteller nach Möglichkeit nachgerüstet werden sollten, sowie das regelmäßige Austauschen bzw. das Reinigen von Filtern und Einwegartikeln.

Ebenfalls thematisiert wird die Wichtigkeit der Unterstützung von Ärzt*innen in der Notaufnahme und Intensivstation bei der Entscheidung zur Aufnahme, Eskalation oder Beendigung einer Behandlung von Patient*innen mit neuromuskulären Erkrankungen und COVID-19 durch neuromuskuläre Spezialist*innen und Pneumolog*innen sowie der generellen Unterstützung der Patient*innen und Angehörigen durch neuromuskuläre Zentren (telefonische Unterstützung, Einrichtung von Videosprechstunden, Gewährleistung der ausreichenden Versorgung heimbeatmeter Patient*innen und Ausstattung der Beatmungssysteme mit Bakterien-Viren-Filter; [52]).

\section{Ausblick}

Nach Überwinden der akuten SARS-CoV-2-Pandemie werden uns im klinischen Alltag neue Symptomkonstellationen wie einerseits Folgezustände und Rehabilitation nach COVID-19-induzierter CIM/CIP, und andererseits ein mögliches chronisches Post-SARS-CoV-2-Syndrom, wie es nach der H1N1-Pandemie 2003 beschrieben wurde, begegnen [53].

Für das akute Outcome unserer Patient*innen wird uns das neue LEOSS Register und für spezielle neuromuskuläre Fragen das „International neuromuscular COVID-19 database registry for COVID-19“ der „University of London“ in absehbarer Zeit datenbasierte Antworten liefern. Aus dem umfangreichen klinischen Datensatz über den Verlauf SARS-CoV-2-Infizierter wird LEOSS durch den kollaborativen, offenen und interdisziplinären Kontext einen wichtigen Beitrag zum Verständnis des Krankheitsbildes und zur evidenzbasierten Entscheidungsfindung auf gesundheitspolitischer Ebene, im Klinikmanagement und in der Individualmedizin leisten.

Literatur

1. Wang D, Hu B, Hu C, Zhu F, Liu X, Zhang J, Wang B, Xiang H, Cheng Z, Xiong Y, Zhao Y, Li Y, Wang X, Peng Z (2020) Clinical characteristics of 138 hospitalized patients with 2019 novel Coronavirus infected pneumonia in Wuhan, China. JAMA 323(11):1061-1069

2. Guan W, Zyi N, Hu Y, Liang W, Ou C, He J, Liu L, Shan H, Lei C, Hui DSC, Du B, Li L, Zeng G, Yuen K-Y, Chen R, Tang C, Wang T, Chen P, Xiang J, Li S, Wang L, Liang Z, Peng Y, Wei L, Liu Y, Hu Y, Peng P, Wang J, Liu J, Chen Z, Li G, Zheng Z, Qiu S, Luo L, Ye C, Zhu S, Zhong N (2020) Clinical characteristics of coronavirus disease 2019 in China. N Engl J Med 382:1708-1720

3. Mao L, Jin H, Wang M, Hu Y, Chen S, He Q, Chang J, Hong C, Zhou Y, Wang D, Miao Y, Li Y, Hu B (2020) Neurologic manifestations of hospitalizes patients with coronavirus disease 2019 in Wuhan, China. JAMA Neurol. https://doi.org/10.1001/jamaneurol. 2020.1127

4. Huang C, Wang Y, Li X, Ren L, Zhao J, Hu Y, Zhang L, Fan G, Xu J, Gu X, Cheng Z, Yu Y, Xia J, Wei Y, Wu W, Xie X, Yin W, Li H, Liu M, Xiao Y, Gao H, Guo L, Xie J, Wang G, Jiang R, Gao Z, Jin Q, Wang J, Cao B (2020) Clinical features of patients infected with 2019 novel coronavirus in Wuhan, China. Lancet 395:497-506

5. Zhou F, Yu T, Du R, Fan G, Liu Y, Xiang J, Wang Y, Song B, Gu X, Guan L, Wie Y, Li H, Wu X, XU J, Tu S, Zhang Y, Chen H, Cao B (2020) Clinical course and risk factors for mortality of adult inpatients with COVID-19 in Wuhan, China: a retrospective cohort study. Lancet 395:1055-1062 
6. LiH, Liu L, Zhang D, XU J, Dai H, Tang N, SuX, Cao B (2020) SARS-CoV-2 and viral sepsis: observations and hypotheses. Lancet. https://doi.org/10.1016/S01406736(20)30920-X

7. AANEM (2020) COVID website. https://www.aanem.org/Practice/COVID-19Guidance. Zugegriffen: 15.05 .20

8. Pinto S et al (2020) Amyotrophic lateral sclerosis and COVID-19 - recommendations to patients and caregivers. https://www.eanpages.org/2020/04/15/amyotrophiclateral-sclerosis-and-covid-19-recommendations-to-patients-and-caregivers/. Zugegriffen: 15.05 .20

9. Guidon AC, Amato AA (2020) Covid-19 and neuromuscular disorders. Neurology. https://doi.org/10.1212/WNL.0000000000009566

10. Supinski GS, Schroder EA, Callahan LA (2020) Mitochondria and critical illness. Chest 157(2):310-322

11. Boya P, Pauleau AL, Poncet D, Gonzalez-Polo RA, Zamzami N, Kroemer G (2004) Viral proteins targeting mitochondria: controlling cell death. Biochim Biophys Acta 1659(2/3):178-189

12. Khan M, Syed GH, Kim SJ, Siddiqui A (2015) Mitochondrial dynamics and viral infections: a close nexus. Biochim Biophys Acta 1853(10 Pt B):2822-2833

13. Zhang L, Qin Y, Chen M (2018) Viral strategies for triggering and manipulating mitophagy. Autophagy 14(10):1665-1673

14. Guzzi PH, Mercatelli D, Ceraolo C, Giorgi FM (2020) Master regulator analysis of the SARS-CoV-2/human interactome. J Clin Med 1 (4):E982

15. Aghagoli G, Gallo Marin B, Soliman LB, Sellke FW (2020) Cardiac involvement in COVID-19 patients: risk factors, predictors, and complications: a review. J Card Surg. https://doi.org/10.1111/jocs.14538

16. Huang I, Lim MA, Pranata R (2020) Diabetes mellitus is associated with increased mortality and severity of disease in COVID-19 pneumonia - A systematic review, meta-analysis, and meta-regression. Diabetes Metab Syndr 14(4):395-403

17. Deutsche Diabetes Gesellschaft Praktische Empfehlungenzum DiabetesManagement bei Patientinnen und Patienten mit einer COVID-19-Erkrankung. www.deutsche-diabetes-gesellschaft.de/fileadmin/Redakteur/Stellungnahmen/ 2020/20200403_Positionspapier_COVID19_final_1.pdf.Zugegriffen: 15.05 .20

18. Wong SH, Lui RN, Sung JJ (2020) Covid-19 and the digestive system. J Gastroenterol Hepatol. https://doi.org/10.1111/jgh.15047

19. Ng YS, Bindoff LA, Gorman GS, Horvath R, Klopstock T, Mancuso M, Martikainen MH, Mcfarland R, NesbittV, Pitceathly RDS, Schaefer AM, Turnbull DM (2019) Consensus-based statements for the management of mitochondrial strokelike episodes. Wellcome Open Res 4:201

20. French JA, Brodie MJ, Caraballo R, Devinsky O, Ding D, Jehi L, Jette N, Kanner A, Modi AC, Newton CR, Patel AA, Pennell PB, Perucca E, Sander JW, Scheffer IE, Singh G, Williams E, Wilmshurst J, Cross JH (2020) Keeping people with epilepsy safe during the Covid-19 pandemic. Neurology. https://doi.org/10.1212/WNL. 0000000000009632

21. De Vries MC, Brown DA, Allen ME, BindoffL, Gorman GS, Karaa A, Keshavan N, Lamperti C, McFarland R, Ng YS, O'Callaghan M, Pitceathly RDS, Rahman S, Russel FGM, Varhaug KN, Schirris TJJ, Mancuso M (2020) Safety of drug use in patients with a primary mitochondrial disease: an international Delphi-based consensus. J Inherit Metab Dis. https://doi.org/10.1002/jimd. 12196

22. Dreher M et al (2020) The characteristics of 50 hospitalized COVID-19 patients with and without ARDS. Dtsch Arztebl Int 117:271-278

23. Dashti-Khavidaki S, Khalili H (2020) Considerations for statin therapy in patients with COVID-19. Pharmacotherapy. https://doi.org/10.1002/phar.2397

24. Jin M, Tong Q (2020) Rhabdomyolysis as potential late complication associated with COVIF19. Emerg Infect Dis. https://doi.org/10.3201/eid2607.200445

25. Yao X et al (2020) In vitro antiviral activity and projection of optimized dosing design of hydroxychloroquine for the treatment of severe acute respiratory syndrome coronavirus 2 (SARS-CoV-1). Clin Infect Dis. https://doi.org/10.1093/cid/ciaa237

26. Boscolli BP (2020) Challenges and cares to promote rational use of chloroquine and hydroxychloroquine in the management of coronavirus disease 2019 (COVID-19) pandemic: a timely review. J Toxicol Environ Health Part B. https://doi.org/10.1080/ 10937404.2020.1752340
27. Magagnoli J et al (2020) Outcomes of hydroxychloroquine usage in United States veterans hospitalized with Covid-19. BMJ. https://doi.org/10.1101/2020.04.16. 20065920

28. Singh AK, Gupta R, Ghosh A, Misra A (2020) Diabetes in COVID-19: Prevalence, pathophysiology, prognosis and practical considerations. Diabetes Metab Syndr 14(4):303-310. https://doi.org/10.1016/j.dsx.2020.04.004

29. Meng L, Qiu H, Wan L, Ai Y, Xue Z, Guo Q, Deshpande R, Zhang L, Meng J, Tong C, Liu H, Xiong L (2020) Intubation and ventilation amid the COVID-19 outbreak: Wuhan's experience. Anesthesiology. https://doi.org/10.1097/ALN. 0000000000003296

30. Vanhorebeek I, Latronico N, van den Berghe G (2020) ICU-acquired weakness. Intensive Care Med 46(4):637-653

31. Arabi YM, Deeb AM, Al-Hameed F, Mandourah Y, Almekhlafi GA, Sindi AA, AlOmari A, ShalhoubS, Mady A, Alraddadi B, Almotairi A, Al Khatib K, Abdulmomen A, Qushmaq I, Solaiman O, Al-Aithan AM, Al-Raddadi R, Ragab A, Al Harthy A, Kharaba A, Jose J, Dabbagh T, Fowler RA, Balkhy HH, Merson L, Hayden FG (2019) Macrolides in critically ill patients with Middle East Respiratory Syndrome. Int J Infect Dis 81:184-190. https:// doi.org/10.1016/j.ijid.2019.01.041

32. Leung TW, Wong KS, Hui AC, To KF, Lai ST, Ng WF, Ng HK (2005) Myopathic changes associated with severe acute respiratory syndrome: a postmortem case series. Arch Neurol 62(7):1113-1117. https://doi.org/10.1001/archneur.62.7.1113

33. Fan E, Cheek F, Chlan L, Gosselink R, Hart N, Herridge MS, Hopkins RO, Hough CL, Kress JP, Latronico N, Moss M, Needham DM, Rich MM, Stevens RD, Wilson KC, Winkelman C, Zochodne DW, Ali NA (2014) An official American Thoracic Society Clinical Practice guideline: the diagnosis of intensive care unit-acquired weakness in adults. Am J Respir Crit Care Med 190(12):1437-1446

34. Medrinal C, Prieur G, Frenoy É, Robledo Quesada A, Poncet A, Bonnevie T, Gravier F, Lamia B, Contal O (2016) Respiratory weakness after mechanical ventilation is associated with one-year mortality — a prospective study. Crit Care 20(1):231

35. Saccheri C, Morawiec E, Delemazure J, Mayaux J, Dubé B, Similowski T, Demoule A, Dres M (2020) ICU-acquired weakness, diaphragm dysfunction and long-term outcomes of critically ill patients. Ann Intensive Care 10(1):1

36. van Gassel RJJ, Baggerman MR, van de Poll MCG (2020) Metabolic aspects of muscle wasting during critical illness. Curr Opin Clin Nutr Metab Care 23(2):96-101

37. van Aerde N, Meersseman P, Debaveye Y, Wilmer A, Gunst J, Casaer MP, Bruyninckx F, Wouters PJ, Gosselink R, van den Berghe G, Hermans G (2020) Five-year impact of ICU-acquired neuromuscular complications: a prospective, observational study. Intensive Care Med. https://doi.org/10.1007/s00134-020-05927-5

38. Pati S, Goodfellow JA, Iyadurai S, Hilton-Jones D (2008) Approach to critical illness polyneuropathy and myopathy. Postgrad Med J 84(993):354-360

39. van der Schaaf M, Beelen A, de Vos R (2004) Functional outcome in patients with critical illness polyneuropathy. Disabil Rehabil 26(20):1189-1197

40. Alberti P, Beretta S, Piatti M, Karantzoulis A, Piatti ML, Santoro P, Viganò M, Giovannelli G, Pirro F, Montisano DA, Appollonio I, Ferrarese C (2020) Guillain-Barré syndrome related to COVID-19 infection. Neurol Neuroimmunol Neuroinflamm. https://doi.org/10.1212/NXI.0000000000000741

41. Toscano G, Palmerini F, Ravaglia S, Ruiz L, Invernizzi P, Cuzzoni MG, Franciotta D, Baldanti F, Daturi R, Postorino P, Cavallini A, Micieli G (2020) Guillain-Barré syndrome associated with SARS-CoV-2. N Eng/ J Med. https://doi.org/10.1056/ NEJMc2009191

42. Zhao H, Shen D, Zhou H, Liu J, Chen S (2020) Guillain-Barrésyndrome associated with SARS-CoV-2 infection: causality or coincidence? Lancet Neurol 19(5):383-384

43. Gutiérrez-Ortiz C, Méndez A, Rodrigo-Rey S, San Pedro-Murillo E, BermejoGuerrero L, Gordo-Mañas R, de Aragón-Gómez F, Benito-Leónet J (2020) Miller Fisher Syndrome and polyneuritis cranialis in COVID-19. Neurology. https://doi.org/ 10.1212/WNL.0000000000009619

44. Dinkin M, Gao V, Kahan J, Bobker S, Simonetto M, Wechsler P, Harpe J, Greer C, Mints G, Salama G, Tsiouris AJ, Leifer D (2020) COVID-19 presenting with ophthalmoparesis from cranial nerve palsy. Neurology. https://doi.org/10.1212/ WNL.0000000000009700

45. International MG/COVID-19 Working Group, Jacob S, Muppidi S, Guidon A, Guptill J, Hehir M, Howard JF Jr, Illa I, Mantegazza R, Murai H, Utsugisawa K, Vissing J, 
Wiendl H, Nowak RJ (2020) Guidance for the management of myasthenia gravis (MG) and Lambert-Eaton myasthenic syndrome (LEMS) during the COVID-19 pandemic. J Neurol Sci 15:412

46. Solé G, Salort-Campana E, Pereon Y, Stojkovic T, Wahbi K, Cintas P, Adams D, Laforet P, Tiffreau V, Desguerre I, Pisella LI, Molon A, Attarian S (2020) FILNEMUS COVID-19 study group. Guidance for the care of neuromuscular patients during the COVID-19 pandemic outbreak from the French RERare Health Care for Neuromuscular Diseases Network. Rev Neurol (Paris). https://doi.org/10.1016/j. neurol.2020.04.004

47. Risitano AM, Mastellos DC, Huber-Lang M, Yancopoulou D, Garlanda C, Ciceri F, Lambris JD (2020) Complement as a target in COVID-19? Nat Rev. https://doi.org/ 10.1038/s41577-020-0320-7

48. Beydon M, Chevalier K, Al Tabaa O, Hamroun S, Delettre A-S, Thomas M, Herrou J, Riviere E, Mariette X (2020) Myositis as a manifestation of SARS-CoV-2. Ann Rheum Dis. https://doi.org/10.1136/annrheumdis-2020-217573

49. aerzteblatt.de (2020) CT erkennt Covid-19 früher als Labortest, aber nicht immer sofort. https://www.aerzteblatt.de/nachrichten/109683/CT-erkennt-Covid-19frueher-als-Labortest-aber-nicht-immer-sofort.Zugegriffen: 15.05.20

50. Leung TW, Wong KS, Hui AC, To KF, Lai ST, Ng WF, Ng HK (2005) Myopathic changes associated with severe acute respiratory syndrome: a postmortem case series. Arch Neurol 62:1113-1117

51. https://www.dgn.org/images/Neuromuskulär_Covid-19_Empf_PB_BS.pdf. Zugegriffen: 15.05 .20

52. Experten der World Muscle Society (2020) Nicht-invasive Heimbeatmung unter der SARS-CoV2-Pandemie - COVID-19-Erkrankung und Menschen mit neuromuskulären Erkrankungen. https://www.dgn.org/neuronews/2uncategorised/3936-nicht-invasive-heimbeatmung-unter-der-sars-cov2pandemie-covid-19-erkrankung-und-menschen-mit-neuromuskulaerenerkrankungen. Zugegriffen: 15.05 .20

53. Moldofsky H, Patcai I (2011) Chronic widespread musculoskeletal pain, fatigue, depression and disordered sleep in chronic post-SARS syndrome; a case-controlled study. BMC Neurol 11:37

54. LEOSS (2020) Statistics. https://leoss.net/statistics/.Zugegriffen: 15.05 .20

55. DIVI (2020) 11.05.2020. https://www.divi.de/register/tagesreport.Zugegriffen: 15.05 .20

\section{Korrespondenzadresse}

\section{Prof. Dr. B. Schoser}

Friedrich-Baur-Institut, Neurologische Klinik und Poliklinik

Ludwig-Maximilians-Universität München

Ziemssenstr. 1, 80336 München, Deutschland

benedikt.schoser@med.uni-muenchen.de

\section{Einhaltung ethischer Richtlinien}

Interessenkonflikt. B. Schoser: Forschungsförderung BMBF (Bundesministerium für Bildung und Forschung), DGM, Sanofi Genzyme, Amicus, Greenovation | Honorar bzw. Kostenerstattung: Kedrion | Berater/interner Schulungsreferent/Gehaltsempfänger o.ä.: Amicus, Audentes, Lupin, Nexien, Sanofi Genzyme. Nicht finanzielle Interessen: Angestellter Oberarzt der Neurologie, Friedrich-Baur-Institut, LMU (Ludwig-Maximilian-Universität) Klinikum München, „EAN Fellow“ (EAN: „European Academy of Neurology“), "EAN educational board member", "EAN rare disease panel board member", „EAN NMJ and myopathies panel board member", DGN Kommissionsvorsitzender NMD und NME, , ERN myopathy board member", „WMS board member teaching course and ICU“. P. Baum gibt an, dass kein finanzieller Interessenkonflikt besteht. Nicht finanzielle Interessen: Oberärztin, Klinik und Poliklinik für Neurologie, Universitätsklinikum Leipzig. M. Boentert gibt an, dass kein finanzieller Interessenkonflikt besteht. K-U. Dillmann: Vortragshonorare; Desitin; Sanofi-Genzyme; Akcea. Nicht finanzielle Inte- ressen: Oberarzt, Klinik für Neurologie; Universitätsklinikum des Saarlandes; Homburg/Saar| Mitgliedschaften: DGM, DGKN (Deutsche Gesellschaft für Klinische Neurophysiologie und funktionelle Bildgebung), DGN | Stellvertretender Landesvorsitzender der Landesgruppe Saar der DGM. A. Emmer gibt an, dass kein Interessenkonflikt besteht. S. Knauss gibt an, dass kein Interessenkonflikt besteht. E. Enax-Krumova gibt an, dass kein finanzieller Interessenkonflikt besteht. Nicht finanzielle Interessen: W1-Stiftungsprofessur für neuronale Regenerationsforschung an der Neurologischen Klinik und Poliklinik des BG Universitätsklinikums, Bergmannsheil gGmbH, RuhrUniversität Bochum, gefördert durch die Deutsche Gesetzliche Unfallversicherung (DGUV) | Mitgliedschaften: Deutsche Gesellschaft für Neurologie e. V., Deutsche Schmerzgesellschaft e. V., Deutsche Migräne- und Kopfschmerzgesellschaft, "International Association for the Study of Pain" (IASP), "Neuropathic Pain Special Interest Group" der IASP, ,Complex regional pain syndrome special interest group" der IASP | Vorstandsmitglied im deutschen Forschungsverbund Neuropathischer Schmerz e. V. J. Grosskreutz gibt an, dass kein finanzieller Interessenkonflikt besteht. Nicht finanzielle Interessen: Leiter des Thüringer Neuromuskulären Zentrums | Oberarzt der Klinik für Neurologie des Universitätsklinikums Jena | Mitglied der DGN | Mitglied der DGM. A-K. Güttsches gibt an, dass kein finanzieller Interessenkonflikt besteht. Nicht finanzielle Interessen: Fachärztin für Neurologie, Neurologische Klinik und Poliklinik, BG-Universitätskliniken Bergmannsheil, Bochum | Mitglied der DGN. K. Hellwig gibt an, dass kein finanzieller Interessenkonflikt besteht. Nicht finanzielle Interessen: Angestellte Neurologin, Klinik und Poliklinik für Neurologie, Katholisches Universitätsklinikum Bochum.

K. Holzapfel gibt an, dass kein finanzieller Interessenkonflikt besteht. Nicht finanzielle Interessen: Oberarzt in Anstellung, Neurologische Klinik und klinische Neurophysiologie Universitätsklinikum Augsburg|Mitglied der DGN, DGKN, DGNB (Deutsche Gesellschaft für neurowissenschaftliche Begutachtung). C. Kornblum: Forschungsförderung: Klinische AMG-Studien (AMG: Arzneimittelgesetz) SPIMM-300, SPIMM-301 (Stealth BioTherapeutics, Inc., USA), POM-03 und ATB200-03 (Amicus Therapeutics, USA), ReDUX4 (Fulcrum Therapeutics, USA); Forschungsförderung mitoNET (BMBF) | Reisekosten, Referentenhonorar: Sanofi Genzyme, Novartis, Santhera, Fulcrum Therapeutics, Amicus Therapeutics, Stealth BioTherapeutics | Berater/interner Schulungsreferent/Gehaltsempfänger o.ä.: Advisory Boards: Stealth BioTherapeutics; Roche Pharma AG. Nicht finanzielle Interessen: Oberärztin der Klinik und Poliklinik für Neurologie, Universitätsklinikum Bonn, Leitung der Sektion Neuromuskuläre Erkrankungen | Mit-Koordinatorin des mitoNET Deutsches Netzwerk für mitochondriale Erkrankungen, BMBF-Förderung translationsorientierter Verbundvorhaben im Bereich der Seltenen Erkrankungen | „Representative" des "Health Care Providers" Universitätsklinikum Bonn, Deutschland (HCP19), Europäisches Referenznetzwerk ERN EURO$\mathrm{NMD}$ |Sprecherin des Zentrums für seltene neurologische Bewegungsstörungen, Epilepsien und Muskelerkrankungen (B-Zentrum) des Zentrums für seltene Erkrankungen Bonn (ZSEB) | Leitung des Muskelzentrums Bonn des Muskelzentrums Nordrhein der DG | Stellvertretende Vorsitzende des mitoNET e. V. (Deutsches Netzwerk für mitochondriale Erkrankungen) | Mitgliedschaften: Deutsche Gesellschaft für Neurologie (DGN); Kommission Neuromuskuläre Erkrankungen und Motoneuronerkrankungen der DGN; Deutsche Gesellschaft für Klinische Neurophysiologie und funktionelle Bildgebung (DGKN); Deutsche Gesellschaft für Ultraschall in der Medizin e.V. (DEGUM e. V.), Sektion Neurologie und Arbeitsgruppe vaskulärer Ultraschall (AvU); Deutsche Gesellschaft für Muskelkranke e. V. (DGM e. V.); mitoNET e. V., Kompetenznetzwerk für Diagnostik und Therapie mitochondrialer Erkrankungen; „World Muscle Society“ (WMS). H. Lehmann: Forschungsförderung: Fa. Genzyme/Sanofi, Fa. Pfizer, Fa. Novartis, Fa. Alnylam | Berater/ interner Schulungsreferent/Gehaltsempfänger o.ä. für Vortragstätigkeit von: Alnylam, Akcea, Biogen, Celgene, CSL Behring, Grifols, Takeda | für Adboards: Alnylam, Akcea, Celgene, CSL Behring, Novartis, Takeda. Nicht finanzielle Interessen: Fachabteilung Neurologie, Klinik und Poliklinik für Neurologie, Universitätsklinikum Köln AoR | Mitgliedschaften: DGN , „Peripheral Nerve Society“. A. Melms: Reisekostenerstattung. Deutsche Myasthenie-Gesellschaft; Deutsche Gesellschaft für Muskelkranke; Berater/interner Schulungsreferent/Gehaltsempfänger o. ä.; Fa. UCB , "Clinical Advisory Board“, „Rozanolixizumab Phase 3 Development Program"; Reisekosten, auf Hono- 
rar verzichtet. Folgende Aktivitäten werden der Vollständigkeit halber genannt und haben keinen thematischen Zusammenhang mit der Publikation: Advisory Boards Fa. Roche; Fa. Biogen; Fa. Sanofi. Nichtfinanzielle Interessen: Niedergelassener Facharzt für Neurologie, Stuttgart Teilzeitbeschäftigung, wiss. Angestellter (5\%) am Universitätsklinikum Tübingen. Mitgliedschaften: Deutsche Gesellschaft für Neurologie (DGN), DGN-Kommission Motoneuron- und Neuromuskuläre Erkrankungen; Deutsche Gesellschaft für klinische Neurophysiologie und funktionelle Bildgebung (DGKN); Deutsche Gesellschaft für Geriatrie (DGG); Gesellschaft für Immunologie; Deutsche Myasthenie-Gesellschaft (DMG, Ärztlicher Beirat) Deutsche Gesellschaft für Muskelkranke (DGM, Ärztlicher Beirat). T. Meyer: Patent/Geschäftsanteile/ Aktien o. ä.: Ambulanzpartner Soziotechnologie APST GmbH, Gründer und Gesellschafter. Nicht finanzielle Interessen: Angestellter Facharzt für Neurologie, Charité - Universitätsmedizin Berlin |DGN, Deutsche Gesellschaft für Neurologie, Mitglied und stellvertretender Vorsitzender der Kommission für Motoneuron- und Muskelerkrankungen |DGM, Deutsche Gesellschaft für Muskelkranke e. V., Mitglied |BMC, Bundesverband Managed Care, Mitglied. S. Petri: Förderung von Forschungsprojekten durch: Deutsche Gesellschaft fürMuskelkranke (DGM), German-Israeli-Foundation (GIF), Bundesministerium für Bildung und Forschung (BMBF) | Honorar und Reisekostenerstattung für Vorträge: Desitin Arzneimittel GmbH, TEVA, Novartis, Biogen | Honorar und Reisekostenerstattung für Beratungstätigkeit: Cytokinetics Inc., Biogen, Roche. Nicht finanzielle Interessen: Angestellte Oberärztin/ Professorin für Neurologie, Neurologische Klinik der Medizinischen Hochschule Hannover | Mitgliedschaft in Fachgesellschaften: DGN, DGKN, DGM. L. Pilgram gibt an, dass kein finanzieller Interessenkonflikt besteht. Nicht finanzielle Interessen: Angestellte Assistenzärztin, Klinik für Innere Medizin 2, Universitätsklinikum Frankfurt. K. Reiners: Honorar oder Kostenerstattung für Tätigkeit als Referent auf Fortbildungsveranstaltungen (ohne thematische Beziehung zu COVID-19): Wörwag Pharma GmbH, Böblingen | Hormosan Deutschland $\mathrm{GmbH}$, Frankfurt | Grifols Deutschland GmbH, Frankfurt. Nicht finanzielle Interessen: Freiberuflicher Facharzt für Neurologie (Consultant) mit Honorartätigkeit am Hermann-Josef-Krankenhaus Erkelenz| Mitgliedschaften: Deutsche Gesellschaft für Neurologie (DGN); Deutsche Gesellschaft für klinische Neurophysiologie und funktionelle Bildgebung (DGKN); Deutsche Gesellschaft für Muskelkranke (DGM); Deutsche Gesellschaft für Neurologische Rehabilitation (DGNR); „Royal Society of Medicine“, London; „European Academy of Neurology ${ }^{\prime} ;$, International Parkinson and Movement Disorder Society“. A. Saak gibt an, dass kein finanzieller Interessenkonflikt besteht. Nicht finanzielle Interessen: Fachärztin, Abteilung Neurologie, Uniklinikum Dresden. J. Schäfer gibt an, dass kein finanzieller Interessenkonflikt besteht. Nicht finanzielle Interessen: Oberarzt der Neurologischen Abteilung, Uniklinikum Dresden | Mitglied DGN, EAN. J. Schmidt: Forschungsförderung von Biogen, CSL Behring, Novartis. Referentenhonorare oder Reisekostenerstattungen von Alnylam, Bayer, Biogen, BioMarin, Biotest, CSL Behring, Euroimmun, Grifols, LFB, Novartis, Octapharma, Pfizer. Beraterhonorare von Alnylam, BioMarin, CSL Behring, Euroimmun, LFB, Novartis, Octapharma. Nicht finanzielle Interessen: Oberarzt der Klinik für Neurologie, Universitätsmedizin Göttingen | Mitglied: DGN, DGM, AAN („American Academy of Neurology“), EAN, Euromyositis, EURO-NMD („European Reference Network Neuromuscular Diseases"), IMACS | Editor: BMC Neurol, JCSM („Journal of Cachexia, Sarcopenia and Muscle"). C. Schneider-Gold gibt an, dass kein finanzieller Interessenkonflikt besteht. M. Schons gibt an, dass kein finanzieller Interessenkonflikt besteht. Nicht finanzielle Interessen: Assistenzarzt, Medizinische Klinik 1, Universitätsklinikum Köln | Mitglied: Deutsche Gesellschaft für Infektiologie.P. P. Urban:Vortragshonorareals Referent der Firmen Abbvie, Alexion, Bial, Daiichi Sankyo, Desitin, Grifols, Zambon. Nicht finanzielle Interessen: Chefarzt Abteilung Neurologie, Asklepios Klinik Barmbek, Hamburg. M. Vorgerd gibt an, dass kein Interessenkonflikt besteht. Nicht finanzielle Interessen: Leitender Oberarzt, Neurologische Klinik und Poliklinik, BG-Universitätskliniken Bergmannsheil, Ruhr-Universität Bochum | Mitgliedschaften:DGN, DGKN P. Young: Honorar bzw. Kostenerstattung von Sanofi-Genzyme, Bioprojet, Medice, Löwenstein-Medical | Berater/interner Schulungsreferent/Gehaltsempfänger o.ä.:Sanofi-Genzyme, Bioprojet. Nicht finanzielle Interessen: Ärztlicher Direktor Medical Park, Bad Feilnbach |Chefarzt Klinik für Neurologie, Medical Park, Bad Feilnbach | Vorsitzen- der Deutsche Gesellschaft Schlafforschung und Schlafmedizin (DGSM) | Vorsitzender des medizinischen-wissenschaftlichen Beirates der DGM. S. Zierz: Honorar oder Kostenerstattung von Fa. Sanofi-Genzyme, Überweisung; Fa. Hormosan, Überweisung | Berater/interner Schulungsreferent/ Gehaltsempfänger o.ä. für Fa. Hormosan, Beratung Myotonia congenita. Nicht finanzielle Interessen: Direktor der Universitätsklinik für Neurologie, Halle/Saale | Mitgliedschaften: DGN, DGM, EAN.

Für diesen Beitrag wurden von den Autoren keine Studien an Menschen oder Tieren durchgeführt. Für die aufgeführten Studien gelten die jeweils dort angegebenen ethischen Richtlinien. 